\title{
Nanoimprint lithography of light trapping patterns in sol-gel coatings for thin film silicon solar cells
}

\author{
Maurits C.R. Heijna ${ }^{* a}$, Jochen Löffler ${ }^{\mathrm{a}}$, Bas B. Van Aken ${ }^{\mathrm{a}}$, Wim J. Soppe ${ }^{\mathrm{a}}$, \\ Herman Borg ${ }^{\mathrm{b}}$, Patrick P.G.J.M. Peeters ${ }^{\mathrm{b}}$ \\ ${ }^{a}$ ECN Solar Energy, Westerduinweg 3, 1755 LE, Petten, the Netherlands; \\ ${ }^{\mathrm{b}} \mathrm{OM} \&$ T B.V., Glaslaan 2, 5616 LW, Eindhoven, the Netherlands
}

\begin{abstract}
For thin-film silicon solar cells, light trapping schemes are of uppermost importance to harvest all available sunlight. Typically, randomly textured TCO front layers are used to scatter the light diffusively in $p-i-n$ cells on glass. Here, we investigate methods to texture the back contact with both random and periodic textures, for use in $n-i-p$ cells on opaque foil. We applied an electrically insulating SiOx-polymer coating on a stainless steel substrate, and textured this barrier layer by nanoimprint. On this barrier layer the back contact is deposited for further use in the solar cell stack. Replication of masters with various random and periodic patterns was tested, and, using scanning electron microscopy, replicas were found to compare well with the originals. Masters with U-grooves of various sub micrometer widths have been used to investigate the optimal dimensions of regular patterns for light trapping in the silicon layers. Angular reflection distributions were measured to evaluate the light scattering properties of both periodic and random patterns. Diffraction gratings show promising results in scattering the light to specific angles, enhancing the total internal reflection in the solar cell.
\end{abstract}

Keywords: thin film solar cells, light trapping, nanoimprint

\section{INTRODUCTION}

Thin film microcrystalline silicon solar cells are an emerging PV technology as a cheaper alternative to the more traditional wafer-based crystalline silicon solar cells since they can be produced using low-cost manufacturing methods [1]. However, the thickness of the absorber layer in these cells $(1 \mu \mathrm{m}$ versus $200 \mu \mathrm{m}$ for wafers $)$ is too thin for the light to be sufficiently absorbed in one pass. As a result, thin film microcrystalline Si solar cells typically need light trapping schemes to enlarge the pathway and to thus enhance the absorption [2], especially in the red and near infrared part of the spectrum [3].

Light scattering in thin film silicon solar cells is usually achieved by nanotextured back reflectors and/or nanotextured transparent conducting oxide (TCO) front electrodes. For $p-i-n$ "superstrate" devices, glass with randomly textured front TCO electrodes is widely used; the textured TCO is typically made by sputtering and texture etching [3]. An alternative to the use of random structures is the use of periodic grating couplers to trap light in solar cells. Photolithographic techniques are required to produce these structures in TCOs [4,5]. These gratings are quite appealing in that they diffract light into the solar cell under specific angles, which may be larger than the angle of total internal reflection, thus trapping the light inside the cell.

In the ECN cell concept for roll-to-roll production of thin film Si solar cells, $n-i-p$ cells are deposited on an opaque flexible substrate consisting of steel foil covered with an electrically insulating barrier layer and a back contact and back reflector (see figure 1). Metal foil is used as substrate to allow for silicon deposition at $200^{\circ} \mathrm{C}$. The insulating barrier layer allows monolithic series connection of solar cells on the steel substrate. In this paper we demonstrate that by nanoimprint lithography the insulating barrier layer can induce light scattering, thus adding extra functionality to this layer. Morphological and optical characterization of various original and replicated random and periodic light scattering structures is presented to demonstrate the versatility of this method to produce substrates for thin film silicon solar cells.

\footnotetext{
*heijna@ecn.nl; phone +31 224 564953; www.ecn.nl
} 


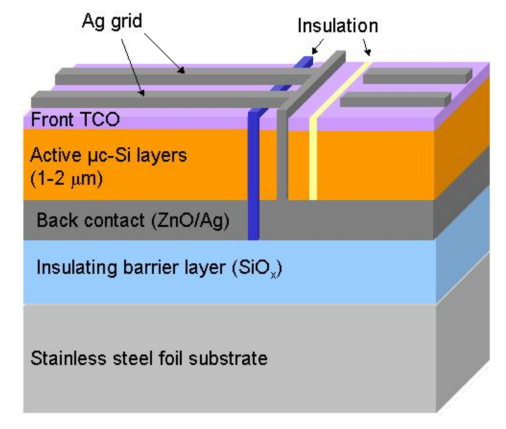

Fig. 1. Schematic representation of the ECN cell concept for thin film silicon solar cells. A flexible metal substrate is used in combination with an insulating barrier layer to allow for monolithic series interconnection. For clarity, the layers are not drawn to scale.

\section{EXPERIMENTAL}

\subsection{Nanoimprint procedure}

In the ECN cell concept, an insulating barrier layer is applied to the stainless steel substrate to electrically separate substrate and solar cell stack in order to allow for series connection of the solar cells. The insulating barrier layer consists of a heat-curing $\mathrm{SiO}_{\mathrm{x}}$-polymer sol-gel coating that can by applied by either roll coating or spray coating. After application, the layer is dried for a short time to such an extend that the surface is dust-dry but the rest of the layer is still plastically deformable. At this point, a texture can be applied to the sol-gel layer by nanoimprint lithography. A master structure of up to $25 \mathrm{~cm}^{2}$ is placed on top of the coated steel foil with its texture towards the barrier layer. The texture is transferred by applying pressure in a hydraulic press with heated anvils, resulting in the inverse of the original morphology. Several different master structures were tested for their replication and optical properties. Due to the (lightly) dried surface of the sol-gel layer, an anti-sticking layer is not required on these masters. Metal masters with diffraction gratings were used for the 1-D periodic structures. These gratings have a groove depth of $300 \mathrm{~nm}$, and periods of 500, 750, and $1000 \mathrm{~nm}$. To create substrates with randomly rough textures, Asahi U-type (SnO:F TCO) and "hot silver" were used. Asahi U-type glass is commercially available, and can be used without any further surface treatment. Hot silver masters can be made by sputtering of silver on glass using high $\left(\sim 400^{\circ} \mathrm{C}\right)$ substrate temperatures. The high substrate temperature results in a rough surface with lateral features in the range of one to several microns, depending on conditions.

\subsection{Characterization of masters and replicas}

To compare the morphology of masters and their replicas, scanning electron microscopy was used. The optical properties of masters and replicas were compared by measuring their haze parameter, and the angular distribution function (ADF) of the reflection.

The wavelength-dependent haze parameter $\mathrm{Hz}$ is the ratio between the diffusely reflected light and the total reflected light, and is given by

$$
H z(\lambda)=\frac{R_{\text {diffuse }}(\lambda)}{R_{\text {total }}(\lambda)},
$$

with $\lambda$ the wavelength, $R_{\text {diffuse }}(\lambda)$ the wavelength-dependent diffuse reflection, and $R_{\text {total }}(\lambda)$ the wavelength-dependent total reflection. The total reflection of a surface is measured using a spectrometer in combination with an integrating sphere to capture all reflected light. The diffuse reflection is measured with the same set-up but the specular reflection is cancelled out by a light trap placed in the integrating sphere.

The haze parameter does not contain any information on the angular distribution function of the diffusely reflected light. For light trapping, high angles of reflection are beneficial as these increase the percentage of light internally reflected at 
the $p$-Si/TCO interface. For reflection angles larger than the angle of total internal reflection, all light will be reflected back into the absorber layer. One can find the angle of total internal reflection $\theta_{t i r}$ by using

$$
\theta_{t i r}=\arcsin \frac{n_{T C O}}{n_{S i}}
$$

Assuming a refractive index at the relevant wavelengths of $600-800 \mathrm{~nm}$ [3] for amorphous Si of $n_{\mathrm{a}-\mathrm{Si}} \approx 3.5$, and for TCO of $n_{\mathrm{TCO}} \approx 2$, the $p$-Si/TCO interface has $\theta_{\text {tir }} \approx 35^{\circ}$, which is taken as an indicative angle for evaluating light scattering substrates.

To measure the ADF of the reflected light of masters and replicas, an optical Goniometer (Instrument Systems GON360) was used in combination with a halogen light source and an Avantes diode array optical spectrometer in the range of 400-1000 nm. Light source and detector are connected to the goniometer by optical fibers. The signal-to-noise ratio of the scattered light of random textures is too low to use the white light source. The ADF of these samples was measured at $632 \mathrm{~nm}$ using a He-Ne laser instead of the halogen lamp.

The main function of the barrier layer is to electrically separate the back contact from the metal substrate. The insulation behaviour should be retained after texturization of the layer. In order to verify the insulating properties, resistance and break-down voltage measured on both flat and textured layers at voltages of 100 up to $1000 \mathrm{~V}$ on surface areas of $4 \times 4$ $\mathrm{mm}^{2}$ and $10 \times 10 \mathrm{~mm}^{2}$.

\section{RESULTS \& DISCUSSION}

\subsection{Random textures}

Using a sample of Asahi U-type SnO:F-coated glass, replicas were made in the barrier layer and compared with the original. Figure 2 shows scanning electron microscopy (SEM) images of randomly textured masters and their replicas. The replica of the Asahi U-type texture shows an inverted and somewhat rounded off structure with respect to its original.

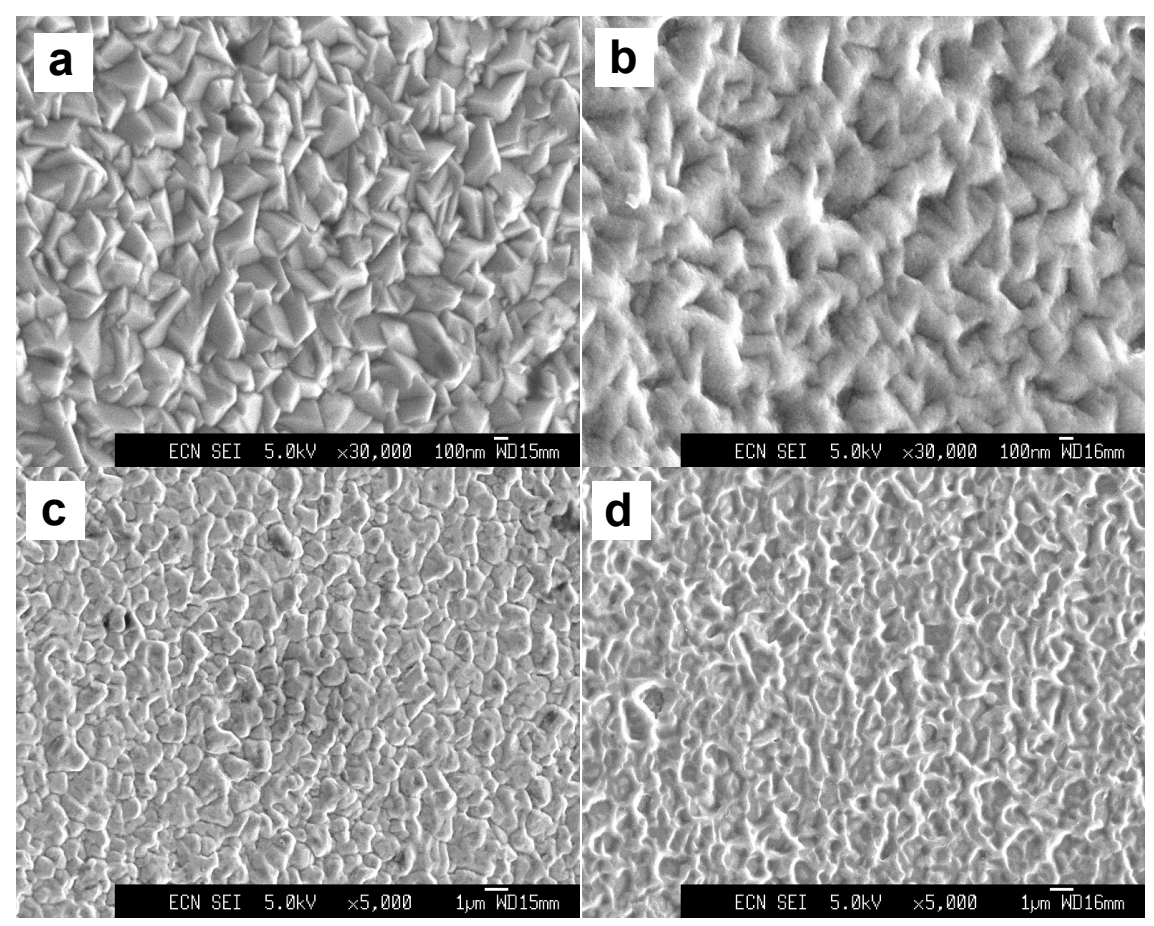

Fig 2. SEM images of randomly textured masters an their replicas in the barrier layer. (a) Asahi U-type SnO:F-coated glass. (b) Replica in the barrier layer. The texture of the replica is inverted with respect to that of the original, and has a $40 \mathrm{~nm} \mathrm{Al}$ 
layer deposited on top of it for optical reflection measurements. (c) Sputtered "Hot Ag" on glass. (d) Replica of the hot $\mathrm{Ag}$ structure in the barrier layer.

.For the optical reflection measurements, both the original Asahi U-type glass and the sol-gel replica were coated with 40 $\mathrm{nm}$ aluminium by e-beam evaporation. Although the structure of the replica is inverted with respect to that of the original, the haze parameter is comparable, showing only a little difference. The haze measurements on the hot silver master and replica, however, show a significant difference (figure 3). Especially at longer wavelengths, in the range of 600 to $1000 \mathrm{~nm}$, the haze of the replica typically is 0.2 lower than the original. The SEM images of the hot Ag original and replica show significant difference in the typical length scale of the morphology (figure 2). The original has large grains in the order of $0.5-3 \mu \mathrm{m}$ with grooves at the grain boundary. Upon replication these grooves become protruding features on top of the surface with typical dimensions of 100-500 $\mathrm{nm}$. The grains replicate as larger flat areas in between these protrusions. Thus, the light scattering properties at longer wavelengths have decreased upon replication.

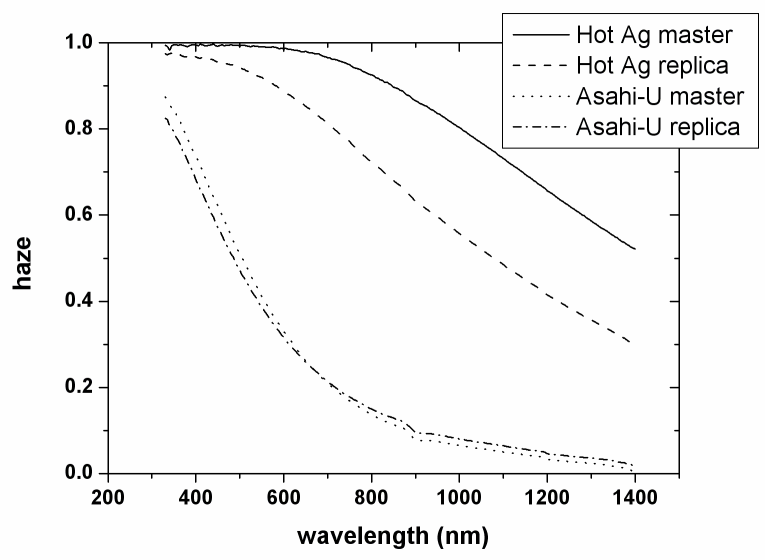

Fig 3. Haze of randomly textured masters and replicas.

To measure the angular distribution function of these randomly textured surfaces a $632 \mathrm{~nm}$ He-Ne laser was used as a light source. In contrast to 1-D periodic gratings, random structures scatter the light with cylindrical symmetry around the surface normal. As a result, the scattered intensity per solid angle is too low for the currently used combination of halogen white light source and spectrometer. The angular distribution at $632 \mathrm{~nm}$ shows a steeper angular dependence of the scattered light for the hot-silver samples than for the Asahi-U samples (figure 4). The scattered intensity at $632 \mathrm{~nm}$ is lower at all angles for the Asahi-U type than for the hot Ag, in accordance with the haze data at this wavelength. Although the haze for the Hot Ag replica is quite high, the largest part of the light is scattered into angles smaller than $\sim 40^{\circ}$. In a solar cell, this substrate is likely to increase the absorption, but total internal reflection can only be obtained for a small fraction of the incident light.

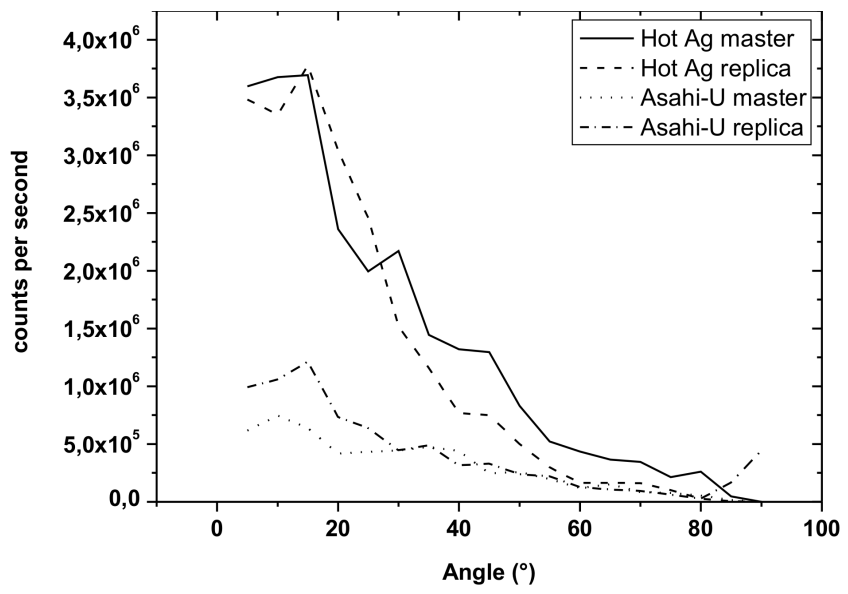


Fig 4. Angular distribution functions of the reflected light of randomly textured masters and their replicas, at a wavelength of $632 \mathrm{~nm}$.

\subsection{Periodic textures}

Figure 5 shows SEM images of the metal masters with one-dimensional diffraction gratings and their replicas. These gratings comprise U-shaped grooves of $300 \mathrm{~nm}$ deep, with three different periods. For all three periods, the replicas compare well with their originals. Experiments are in progress to compare masters and replicas more quantitatively by using atomic force microscopy.

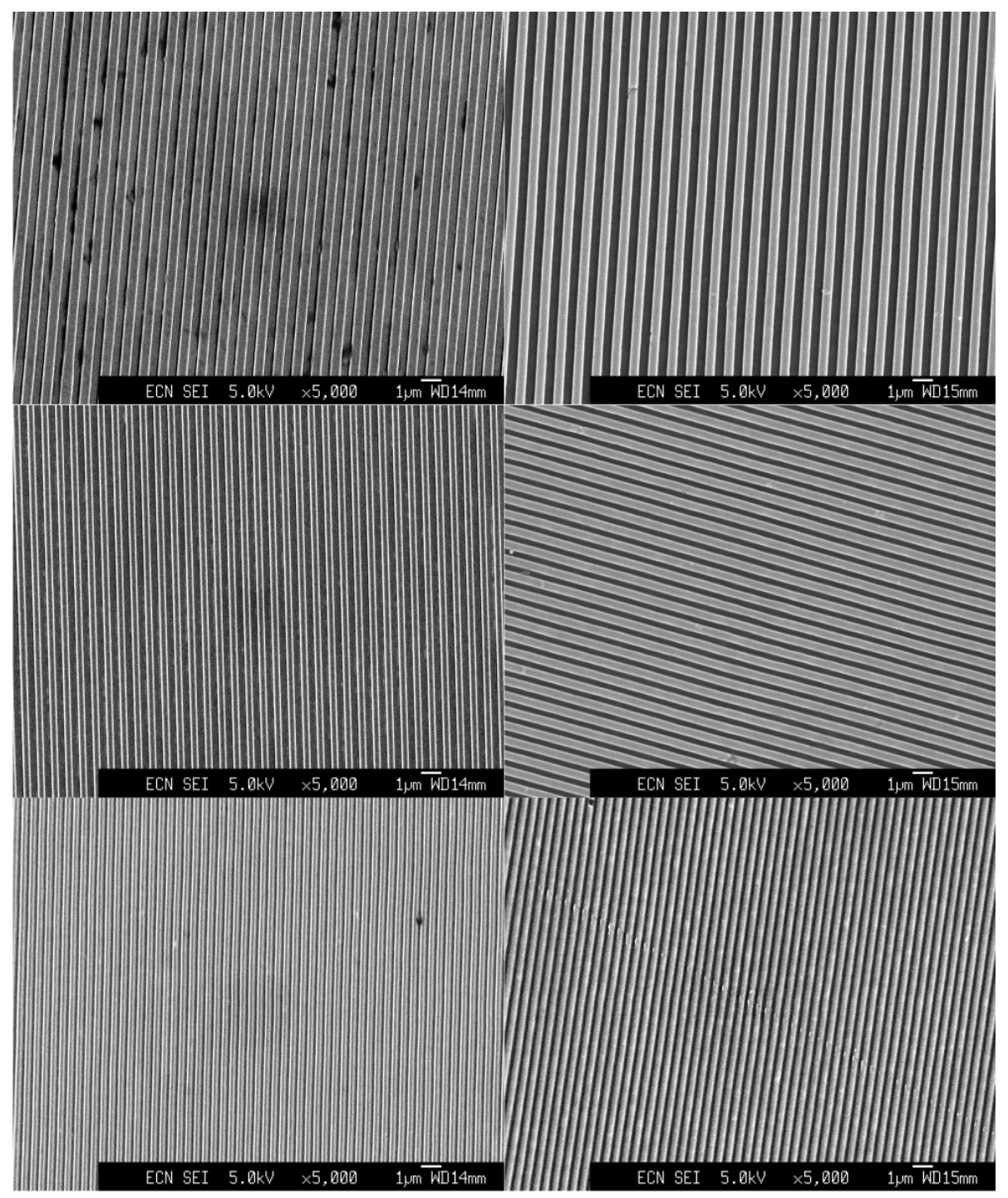

Fig 5. SEM images of periodically structured metal masters (left column) and their respective replicas in the sol-gel layer (right column). From top to bottom the periods of the grating are $1000 \mathrm{~nm}, 750 \mathrm{~nm}$, and $500 \mathrm{~nm}$. The scale bar in each figure represents $1 \mu \mathrm{m}$.

Haze measurements on the masters and Al-covered replicas show that for wavelengths larger than the period of the grating no diffraction occurs. At wavelengths equal to the period of the grating, the angle of diffraction is $90^{\circ}$, following the diffraction formula given by

$$
\sin \theta=m \frac{\lambda}{p}+\sin \theta_{i},
$$


with $\theta$ the angle of diffraction, $\lambda$ the wavelength, $m$ the diffraction order, $p$ the period of the grating, and $\theta_{i}$ the angle of incidence. For higher wavelengths no diffraction occurs and all light is reflected specularly. Qualitatively the haze of the masters and Al-covered replicas match, but quantitatively there are deviations due to the difference in material.
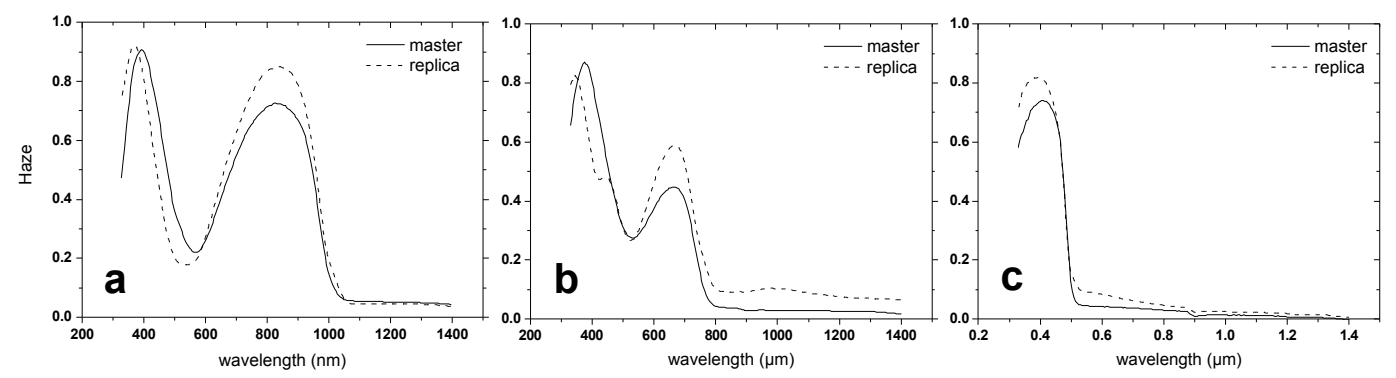

Fig 6. Haze measurements on masters and replicas with diffraction gratings of (a) $1000 \mathrm{~nm}$, (b) $750 \mathrm{~nm}$, and (c) $500 \mathrm{~nm}$.

Figure 7 shows the ADF of the reflected light for the replicas of the diffraction gratings relative to the intensity of the incident light. As can be expected, the wavelength-dependent angle of diffraction follows the theoretical relation of equation 3 for all three replicas. For the $1000 \mathrm{~nm}$ period also the second order diffraction $(\mathrm{m}=2)$ is visible, for the other two gratings the second order diffraction peaks fall outside the wavelength range of the detector and source. For both the $750 \mathrm{~nm}$ and $1000 \mathrm{~nm}$ grating the lower intensity of diffracted light around $500 \mathrm{~nm}$ is reflected in the reduced haze around this wavelength. The diffracted intensities of the gratings correspond well to the haze measurements. For instance, at wavelengths around $800 \mathrm{~nm}$ the ADF shows the diffraction peaks to have approximately $40 \%$ of the incident light intensity. A similar ADF is present for negative angles, thus a total of $80 \%$ of the incident light is diffracted at an angle of $\pm 60^{\circ}$ at a wavelength of $800 \mathrm{~nm}$, in correspondence with the haze at this wavelength (dashed line in figure 6a). For the estimated angle of total internal reflection of $35^{\circ}$, the diffraction equation (eq. 3) shows that the grating with $1000 \mathrm{~nm}$ period has the most optimal diffraction for use with amorphous silicon and at longer wavelengths for microcrystalline silicon. The experimental ADF shows this grating to have the highest diffraction intensity of the gratings tested in this paper. For amorphous silicon, the grating with $750 \mathrm{~nm}$ period may also be used as its ADF approximately matches the relevant wavelength range of $600-800 \mathrm{~nm}$, but has significantly lower diffracted intensity than the $1000 \mathrm{~nm}$ period in this range. The $500 \mathrm{~nm}$ grating however is not suited for use as a substrate as above $500 \mathrm{~nm}$ all light is reflected specularly.
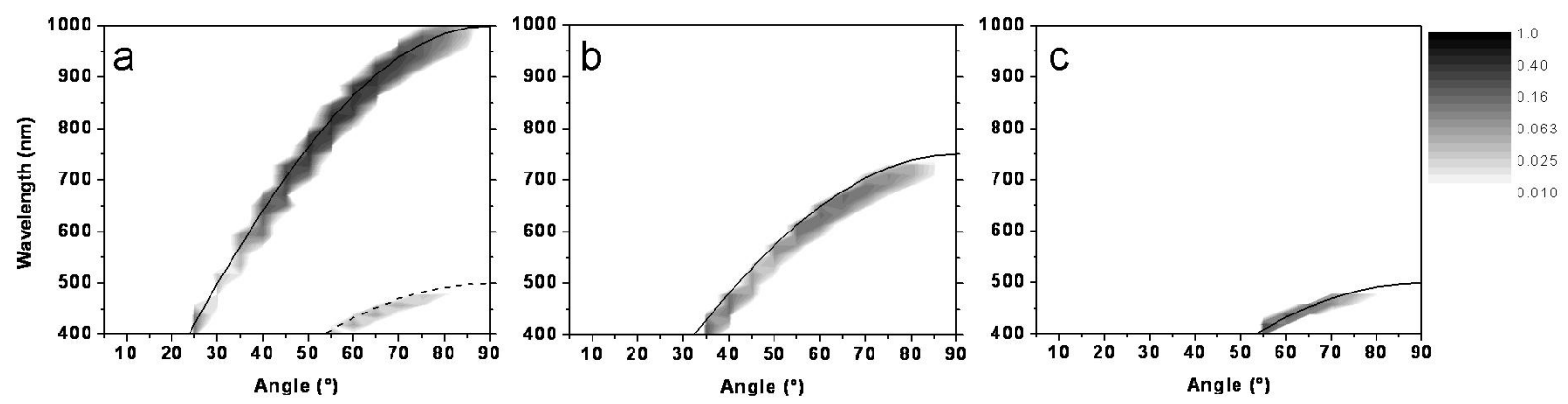

Fig 7. Angular distribution functions of reflection for replicated diffraction gratings at perpendicular incident light, showing intensity relative to the incident light intensity $(=1)$ on a logarithmic scale. The step-wise shape of the diffracted intensity is an artifact of the $5^{\circ}$ steps in the detector angle. Panel (a) shows the ADF for the $1000 \mathrm{~nm}$ period grating, with the theoretical relation between angle and wavelength of the diffraction peak indicated by the solid (first order) and dashed (second order) lines. Panel (b) shows the distribution for the $750 \mathrm{~nm}$ period, and panel (c) for the $500 \mathrm{~nm}$ grating.

\subsection{Electrical properties}

A periodic structure consisting of a mixture of submicron grooves was used to test the electrical insulation of textured barrier layers with various feature sizes in one experiment. The flat reference sample was part of the same $10 \mathrm{by} 10 \mathrm{~cm}^{2}$ piece of coated foil, in order to cancel out any differences in layer thickness between barrier layer depositions. Table 1 
shows the average breakdown voltage and resistance value. Although texturing the foil reduces the resistance of the layer typically by a factor 4 , the breakdown voltage remains higher than $1000 \mathrm{~V}$. Thus, the electrical properties remain well within the limits for application in the ECN solar cell concept.

Table 1. Resistance and breakdown voltage of flat and textured barrier layers for $0.16 \mathrm{~cm}^{2}$ and $1 \mathrm{~cm}^{2}$ contact pads.

\begin{tabular}{|c|c|c|c|}
\hline & $\begin{array}{l}R_{100 \mathrm{~V}} \\
(G \Omega)\end{array}$ & $\begin{array}{l}R_{500 \mathrm{~V}} \\
(G \Omega)\end{array}$ & $\begin{array}{c}\text { V }_{\text {breakdown }} \\
\text { (V) }\end{array}$ \\
\hline Flat, $0.16 \mathrm{~cm}^{2}$ & 165 & 93 & $>1000$ \\
\hline Flat, $1 \mathrm{~cm}^{2}$ & 89 & 33 & $>1000$ \\
\hline$\overline{\text { Textured, } 0.16 \mathrm{~cm}^{2}}$ & 40 & 20 & $>1000$ \\
\hline Textured, $1 \mathrm{~cm}^{2}$ & 10 & 4 & $>1000$ \\
\hline
\end{tabular}

\section{CONCLUSION}

Light trapping structures are of utmost importance for thin-film microcrystalline silicon solar cells. We have demonstrated that by nanoimprint random and periodic textures can be replicated in sol-gel barrier layers without loss of the insulating functionality of these layers. The angular distribution of reflected light suggest that periodic grating patterns of 750 to $1000 \mathrm{~nm}$ are suited to enhance the light trapping in thin film Si solar cells. Nanoimprint lithography of sol-gel layers offers a versatile method to replicate light-trapping structures of any choice for solar cell sub- and superstrate production.

\section{ACKNOWLEDGEMENTS}

This work has been financially supported by the Dutch Ministry of Economic Affairs (Project No. EOSLT04029).

\section{REFERENCES}

[1] Maycock, P. and Bradford, T., "PV Technology, Performance, and Cost, 2007 Update", Prometheus Institute for Sustainable Development and PV Energy Systems, 2007.

[2] Deckman, H. W., Roxlo, C. B. and Yablonovich, E., "Maximum statistical increase of optical absorption in textured semiconductor films", Opt. Lett. 8, 491-493 (1983).

[3] Müller, J., Rech, B., Springer, J. and Vanecek, M., "TCO and light trapping in silicon thin film solar cells", Solar Energy 77, 917-930 (2004).

[4] Senoussaoui, N., Krausse, M., Müller, J., Bunte, E., Brammer, T. And Stiebig, H., "Thin-film solar cells with periodic grating coupler“, Thin Solid Films 451-452, 397-401 (2004)

[5] Eisele, C., Nebel, C.E. and Stutzmann, M., "Periodic light coupler gratings in amorphous thin film solar cells“, J. Appl. Phys. 89, 7722-7726 (2001) 